\title{
GIARDIOSE E CRIPTOSPORIDIOSE EM CRECHES NO MUNICÍPIO DE CAMPINAS, SP
}

\author{
Regina Maura B. Franco e Nelson da S. Cordeiro
}

\begin{abstract}
Estudou-se as prevalencias de Giardia duodenalis e Cryptosporidium parvum, conforme sexo e faixa etária, em crianças de $2-60$ meses $(n=310)$, atendidas em 8 creches. Giardiose ocorreu em $42(13,5 \%)$ das crianças e foi mais frequente na faixa etária de 19-24 meses, embora crianças nos primeiros seis meses de vida apresentassem amostras fecais positivas. Teste estatístico nãa revelou associação entre sexo e parasitismo $(p>0,05)$. C. parvum foi encontrado em 20 crianças $(6,4 \%)$ com maior frequência no grupo de 7-12 meses. Crianças entre 25-30 meses e acima de 36 meses não apresentaram oocistos nas fezes. Oocistos foram detectados em 4 crianças na faixa etāria de 0-6 meses. Não boute significância estatîstica entre criptosporidiose e sexo ( $p$ $=0,09)$ e, entre creches $(p=0,068)$. Ocorreu associação entre idade e a presença do coccídio $(p=0,004)$. Abordou-se os aspectos epidemiológicos por meio de inquérito familiar; exames parasitológicos entre contatos dos casos-indices e dos animais presentes no domicílio. Prevalencia de C. parvum foi maior quando pelo menos um dos pais era profissional da Área de Saúde $(73,6 \%$ positivas), havia relato de doença concomitante (52,6\% positivas) e diarréia era o principal sintoma por ocasiâo de exame fecal (78,9\% positinas). Exames de fezes dos animais domésticos foram negativos para estes dois parasitos.
\end{abstract}

Palauras-chates: Criptosporidiose. Giardiose. Crianças. Creches.

$O$ aumento da participação da mulher no mercado de trabalho foi acompanhado por mudanças no cuidado infantil, antes prestado no âmbito do lar, agora delegado às creches. Esta situação comportamental trouxe como conseqüência implicações epidemiológicas profundas: crianças em idade pré-escolar estão, hoje em dia, sujeitas à aquisição precoce de agentes infecciosos ${ }^{21}$. Características da própria criança como a imaturidade do sistema imunológico infantil, a fase oral de exploração, a incontinência fecal, os hábitos de higiene ainda em formação e, das creches, como o grande número de crianças alojadas em locais fechados, condições de higiene inadequadas e atendentes mal treinados propiciam maior risco de exposição.

As infecções causadas por parasitos, considerados os danos que ocasionam no desenvolvimento físico e mental na infância,

\footnotetext{
Laboratório de Protozoologia, Departamento de Parasitologia do Instituto de Biologia, Universidade Estadual de Campinas, Campinas, SP.

Endereço para correspondência: Dr Regina Maura B. Franco. Laboratório Protozoologia/IB/UNICAMP. Caixa Postal 6109, 13081-970 Campinas, SP. Fax: (019) 239-3124. Recebido para publicação em 23/10/95.
}

passaram a representar um importante problema no contexto das creches. Protozoários patogênicos como Cryptosporidium parvum, Giardia duodenalis ( $G$. lamblia ou $G$. intestinalis), Entamoeba histolytica, Isospora belli ou comensais como Endolimax nana, Entamoeba coli, Iodamoeba bütscblii e outros, de patogenicidade discutivel como Dientamoeba fragitis ocorrem em crianças e podem estar relacionados com grau variável de desconforto abdominal ${ }^{15}$. Dados da literatura mostram que Giardia duodenalis e Cryptosporidium parvum vem apresentando ocorrência crescente em creches que atendem crianças na faixa etária de 0 a 5 anos de idade 7172632 em vários países. G. duodenalis é um protozoário flagelado cujo papel patogênico foi reconhecido desde que surtos de giardiose foram descritos nas décadas de 60 e $70^{25}$. C. parvum é um protozoário intestinal cuja importância foi demonstrada a partir dos relatos dos primeiros casos de AIDS, após 1976. As manifestações clínicas ocorrem de acordo com o estado imunológico do hospedeiro, sendo a diarréia o principal sintoma ${ }^{1+}$.

$G$. duodenalis e C. parvum apresentam aspectos epidemiológicos em comum: ambos podem provocar infecções assintomáticas, permanecendo transmissiveis enquanto perdurar 
Franco RMB, Cordeiro NS. Giardiose e criptosporidiose em crecbes no município de Campinas, SP. Revista da Sociedade Brasileira de Medicina Tropical 29:585-591, nov-dez, 1996.

a excreção de formas resistentes nas fezes; os cistos e oocistos são capazes de sobreviver no meio externo e continuarem infectantes por meses, conforme as condições de umidade e temperatura; a infecção experimental pode ser estabelecida mesmo com doses mínimas de cistos e oocistos - ao redor de 10 cistos para G. duodenalis ${ }^{27}$ e de 1 a 5 oocistos no caso de C. parvum ${ }^{8}$ - podem ser transmitidos pela água de beber ${ }^{14}$ embora, nos países em desenvolvimento, o contato pessoa-à-pessoa pareça ser a principal via de transmissão ${ }^{7}$ e, finalmente, apresentam um pico de ocorrência em crianças na faixa etária entre 1-5 $\operatorname{anos}^{133133}$.

No Brasil, Oliveira ${ }^{2-1}$ relatou que $10 \%$ dos episódios diarréicos agudos na infância estão relacionados aos protozoários intestinais. Oocistos de C. parvum tem sido demonstrado nas fezes de crianças imunocompetentes, portadoras de diarréia ${ }^{20}{ }^{37}$ e em crianças apresentando imunodeficiências ${ }^{12}$. Giardiose tem sido assinalada em crianças freqüentando creches nas cidades de São Paulo ${ }^{35}$ e Aracajú.

Em Campinas, SP, dados fornecidos pela Secretaria Municipal de Educação revelam que a proporção de crianças freqüentando creches é bastante alta. Neste estudo, procurou-se averiguar com que freqüência ocorreram infecçōes por Giardia duodenalis e Cryptosporidium parvum entre lactentes com 2-60 meses de idade que são atendidas em creches, com o propósito de comparação com outras localidades geográficas.

\section{MATERIAL E MÉTODOS}

Entre agosto de 1990 e junho de 1993 foram realizados exames coproparasitológicos em 310 crianças, com idade entre $2-60$ meses, de ambos os sexos, matriculadas em 8 creches localizadas no Distrito de Barão Geraldo, Município de Campinas, SP.

Foram colhidas, em dias alternados, três amostras de fezes por criança, apresentando ou não diarréia. Cada amostra, preservada em formalina $10 \%$, foi submetida à técnica de Ritchie $^{28}$ e à concentração por centrífugosedimentação em éter-PBS ${ }^{36}$. Esfregaços do concentrado foram corados pelo método de Ziehl-Neelsen modificado ${ }^{16}$ para determinar a presença de oocistos de C. parvum.

Crianças que eliminaram oocistos de $C$. parvum nas fezes, constituíram os casos-índices que serviram para identificar as pessoas que foram submetidas a estudos mais completos (aplicação de questionário-padrão em inquéritofamiliar). Animais domésticos (3 cães e 1 gato), quando presentes no domicílio, tiveram 12 amostras fecais examinadas pelo método de swab retal, após coloração de Ziehl-Neelsen modificada ${ }^{16}$.

Medidas dos oocistos foram obtidas com o auxílio de ocular calibrada com lâmina micrométrica e, considerados como positivos os espécimens com diménsões ao redor de 46um e morfologia compativel com oocistos de C. parvum.

Os resultados obtidos foram submetidos à análise estatística, utilizando-se o teste do $\chi^{2}$ de Pearson, com nível de significância de $\mathrm{p} \leq 0,05$, para tabela de contingencia $2 \times 2$.

\section{RESULTADOS}

No presente estudo, foram analisadas fezes de 310 crianças ( 165 do sexo masculino e 145 do sexo feminino), em um total de 8 creches examinadas. Dados da capacidade de atendimento das creches, número e nível sócio-econômico das crianças incluídas no estudo são apresentados na Tabela 1. Todas as creches eram servidas por água encanada e tratada, fornecida pela SANASA (Sociedade de Abastecimento de Água e Saneamento S/A) e a coleta de lixo era realizada diariamente pela Prefeitura Municipal de Campinas (PMC).

Tabela 1 - Número de crianças examinadas condição sócioeconônica e capacidade de atendimento em oito crecbes no municipio de Campinas. SP (1990-93)

\begin{tabular}{cccc}
\hline Creche & $\begin{array}{c}\text { Capacidade de } \\
\text { atendimento }\end{array}$ & $\begin{array}{c}\text { Nível sócio- } \\
\text { economico }\end{array}$ & $\begin{array}{c}\text { No de crianças } \\
\text { examinadas }\end{array}$ \\
\hline 1 & 60 & $>10$ & 28 \\
2 & 180 & $>10$ & 61 \\
3 & 25 & $>10$ & 14 \\
4 & 30 & $5-10$ & 25 \\
5 & 50 & $>10$ & 25 \\
6 & 100 & $>10$ & 33 \\
7 & 120 & $<5$ & 43 \\
8 & 240 & $5-10$ & 81 \\
\hline
\end{tabular}

"SM= Salário minimo.

Oocistos de C. parvum foram detectados em 20 crianças $(6,4 \%)$ e cistos de $G$. duodenalis em $42(13,5 \%)$. Exame fecal negativo, em pelo menos uma ocasião, concomitante à ocorrência de diarréia, foi observado em 34 crianças $(10,9 \%)$.

Giardia duodenalis. Nas Tabelas 2 e 3, são apresentados os dados sobre o parasitismo 
Franco RMB, Cordeiro NS. Giardiose e criptosporidiose em crecbes no municipio de Campinas, SP. Revista da Sociedade Brasileira de Medicina Tropical 29:585-591, nov-dez, 1996.

relacionado à $G$. duodenalis. Entre as 165 crianças do sexo masculino examinadas, 22 $(7,1 \%)$ foram positivas e, para o sexo feminino, notou-se que das 145 analisadas, $20(6,4 \%)$ apresentavam cistos nas fezes. O tratamento estatístico não revelou diferença significativa entre os sexos para giardiose $\left(\chi^{2}=0,014\right.$; $\mathrm{p}=0,906 ; \mathrm{OR}=0,542-1,995, \mathrm{IC} 95 \%) \mathrm{mas}$ apontou diferenças entre as idades $\left(\chi^{2}=13,007\right.$ e $p=0,043)$ e creches $\left(\chi^{2}=15,968 ; p=0,025\right)$.

Embora crianças em todas as faixas etárias apresentassem parasitismo por $G$. duodenalis, inclusive aquelas nos primeiros seis meses de vida, a maior taxa de positividade foi encontrada no grupo com idade entre 19-24 meses.

Cryptosporidium parvum. Vinte crianças $(6,4 \%)$ apresentaram oocistos nas fezes, sendo 7 crianças do sexo masculino e 13 do sexo feminino. O teste do qui-quadrado revelou ausência de significância entre parasitismo pelo protozoário e sexo $\left(\chi^{2}=2,853 ; \mathrm{p}=0,09\right.$; $\mathrm{OR}=0,862-5,733$, IC 95\%) e também demonstrou que a diferença não é significativa $\left(\chi^{2}=13,156\right.$; $\mathrm{p}=0,068$ ) entre as creches (Tabela 3). No

Tabela 2 - Distribuição da prevalência de cistos de Giardia duodenalis e de oocistos de Cryptosporidium parvum, respectivanente, em crianças de diferentes idades. freqüentando creches em Campinas, SP (1990-93).

\begin{tabular}{|c|c|c|c|c|c|c|c|c|}
\hline \multirow[t]{2}{*}{ Exame fecal } & \multicolumn{8}{|c|}{ Idade (em meses) } \\
\hline & $0-6$ & $7-12$ & $13-18$ & $19-24$ & $25-30$ & $31-36$ & $>36$ & Total \\
\hline \multicolumn{9}{|c|}{ Giardia duadenalis } \\
\hline negativo & 73 & 48 & 35 & 35 & 22 & 29 & 24 & $266^{*}$ \\
\hline positivo & 4 & 4 & 6 & 12 & 5 & 5 & 5 & $41^{*}$ \\
\hline$(\%)$ & $(1.3)$ & (1.3) & $(1.9)$ & $(13.9)$ & $(1,6)$ & $(1.6)$ & $(1,6)$ & $(13,3)$ \\
\hline \multicolumn{9}{|l|}{ C. parnum } \\
\hline$(\%)$ & $(23.7)$ & $(13.6)$ & $(12.7)$ & $(14,3)$ & $(8.7)$ & $(10.7)$ & $(9.4)$ & $(93,4)$ \\
\hline positivo & 4 & 10 & 2 & 3 & 0 & 1 & 0 & $20^{*}$ \\
\hline$(\%)$ & $(1,3)$ & $(3.2)$ & $(0,6)$ & $(0,9)$ & $(0,0)$ & $(0.3)$ & $(0,0)$ & $(6,4)$ \\
\hline Total & 77 & 52 & 41 & 47 & 27 & 34 & 29 & $307^{*}$ \\
\hline$\%$ & $(25,0)$ & $(16.9)$ & $(13.3)$ & $(15.3)$ & $(8,7)$ & $(11,0)$ & $(9,4)$ & $(100,0)$ \\
\hline
\end{tabular}

*3 crianças tiveram a idade indeterminada.

Tabela 3 - Pretalência de Giardia duodenalis e Cryptosporidium parum en criancas na faixa etária de 2-60 meses, en oito crecbes no municipio de Campinas. SP (1990-93).

\begin{tabular}{|c|c|c|c|c|c|c|c|c|c|}
\hline \multirow[t]{3}{*}{ Creche } & \multicolumn{8}{|c|}{ Exame fecal } & \multirow[b]{3}{*}{ Tota } \\
\hline & \multicolumn{4}{|c|}{ Giardia duadenalis } & \multicolumn{4}{|c|}{ Coptosporiditum parum } & \\
\hline & $f(-)^{*}$ & $\%$ & $f(+)^{* *}$ & $\%$ & $f(-)$ & $\%$ & $f(t)$ & $\%$ & \\
\hline 1 & 18 & 64.2 & $\overline{10}$ & 35.7 & 27 & 96.4 & 1 & 3.5 & 28 \\
\hline 2 & 54 & 88.5 & 7 & 11.4 & 53 & 86.8 & 8 & 13,1 & 61 \\
\hline 3 & 12 & 85.7 & 2 & 14.2 & 14 & $\begin{array}{c}100,0 \\
0\end{array}$ & 0 & 0,0 & 14 \\
\hline 4 & 22 & 88.0 & 3 & 12,0 & 25 & $\begin{array}{c}100.0 \\
0\end{array}$ & 0 & 0.0 & 25 \\
\hline 5 & 22 & 88.0 & 3 & 12,0 & 25 & $\begin{array}{c}100.0 \\
0\end{array}$ & 0 & 0,0 & 25 \\
\hline 6 & 32 & 96.9 & 1 & 3.0 & 33 & $\begin{array}{c}100.0 \\
0\end{array}$ & 0 & 0,0 & 33 \\
\hline 7 & 39 & 90.6 & 4 & 9.3 & 40 & 1.9 & 3 & 6.9 & 43 \\
\hline 8 & 69 & 85.1 & 12 & 14.8 & 73 & 90.1 & 8 & 9,8 & 81 \\
\hline Total & 268 & 86.4 & 42 & 13.3 & 290 & 93.5 & 20 & 6,4 & 310 \\
\hline
\end{tabular}

* $f(-)=$ freqüencia de exame fecal negativo; ${ }^{* *} f(+)=$ freqüência de exame fecal positivo

entanto, quando se considerou a estratificação etária, encontrou-se significância $\left(\chi^{2}=18,824\right.$; $\mathrm{p}=0,004$ ) (Tabela 2).

Das 8 creches envolvidas no estudo, 4 não apresentaram crianças portadoras de criptosporidiose; em duas creches, foram detectadas 8 crianças positivas (Tabela 3 ).
Crianças nas faixas etárias entre 25-30 meses e acima de 36 meses não apresentaram parasitismo por $C$. parvum enquanto que oocistos foram detectados nos primeiros meses de vida (4 crianças na faixa etária entre 0-6 meses), acentuando-se a ocorrência deste protozoário em lactentes com idade entre 7-12 meses (Tabela 2). 
Franco RMB, Cordeiro NS. Giardiose e criptosporidiose em creches no municipio de Campinas, SP. Revista da Sociedade Brasileira de Medicina Tropical 29:585-591, nov-dez, 1996.

Em relação à consistência das fezes, 17 $(5,4 \%)$ crianças apresentaram diarréia na ocasião em que o esfregaço fecal foi positivo para C. parum, sendo apenas $0,6 \%$ a taxa de infecção assintomática por este coccídio. Quando considerado parasitismo por $G$. duodenalis, 10 $(3,2 \%)$ lactentes apresentaram diarréia e exame fecal positivo. Ausência de diarréia, na presença deste protozoário foi observada em $24(7,7 \%)$ crianças. Parasitismo concomitante por $G$. duodenalis e C. parvum foi observado em duas crianças.

Outros parasitos intestinais ocorreram em 5 crianças, a saber: Ascaris lumbricoides em 1 lactente $(0,3 \%)$, Entamoeba coli em $1(0,3 \%)$, Chilomastix mesnili em $1(0,3 \%)$ e Tricburis tricbiura em $2(0,6 \%)$.

Em relação às características epidemiológicas das crianças portadoras de C. parvum verificou-se que a prevalência do protozoário foi maior quando: pelo menos um dos pais era profissional da Area de Saúde (73,6\% positivas e $26,3 \%$ negativas), não havia presença de animais domésticos no domicílio (31,5\% positivas e $68,4 \%$ negativas), havia telato de doença concomitante $(52,6 \%$ positivas e $42,1 \%$ negativas) e diarréia era o principal sintoma relatado por ocasião da coleta da amostra fecal (78,9\% positivas e $21,0 \%$ negativas).

Dos 65 indivíduos que mantiveram contato com as crianças com criptosporidiose, 31 $(47,6 \%)$ eram adultos (11 familiares e 20 funcionários das creches) e $34(52,3 \%)$ crianças (33 ocupavam o mesmo quarto na creche e 1 contato familiar). Positividade para C. paruum somente foi observada em $12,1 \%(4+/ 33)$ das crianças que compartilhavam o mesmo quarto e, entre os funcionátios, apenas foram detectados ancilostomídeos $(4+/ 20)$. Foram examinados 4 animais domésticos ( 3 cães e 1 gato), todos com resultado negativo.

\section{DISCUSSÃO}

Na última década, vários pesquisadores voltaram sua atenção para a ocorrência de giardiose e de criptosporidiose em crianças freqüentando creches ${ }^{23717} \mathrm{e}$, reconheceram que as creches tornaram-se ambientes únicos do ponto de vista epidemiológico onde as crianças estão sujeitas à maior exposição às doenças infecto-contagiosas ${ }^{21}$.

A prevalência de $G$. duodenalis observada no presente estudo foi ligeiramente menor se comparada as taxas de 16\%-38\%, encontradas por outros autores 73262933 . No Brasil, foram assinaladas taxas bem elevadas para este protozoário ${ }^{35}$. Em diferentes levantamentos, dependendo dos métodos utilizados e da populaçâo estudada, a prevalência de $G$. duodenalis varia grandemente. Uma possivel razão para a discrepância dos nossos resultados, poderia estar relacionada ao perfil sócioeconômico das crianças pesquisadas (superior à $10 \mathrm{SM}$ ) e também pelo fato de terem sido incluídas na amostragem crianças nos primeiros meses de vida.

Giardiose é, atualmente, um problema comum em creches onde freqüentemente o contato pessoa-à-pessoa é potencializado e as medidas higienicas adequadas são difícies de serem mantidas. Dados da literatura ${ }^{73}$ mostram que nos primeiros nove meses de vida, a ocorrência de $G$. duodenalis é baixa mas sofre um aumento substancial durante o segundo e terceiro anos de vida, quando a criança inicia o treinamento da higiene, o que também foi observado neste estudo. De acordo com Bartllet e cols ${ }^{*}$ as diferenças comportamentais entre crianças de $0-12$ meses e aquelas com 19-24 meses poderiam influenciar os diferentes níveis de exposição ao agente infeccioso. Visto que a diferença entre as prevalências de giardiose nas creches estudadas foi estatisticamente significante, pode-se deduzir que fatores tais como características da creche, não detectadas neste estudo, poderiam ter influenciado os resultados obtidos.

Addiss e cols² demonstraram que o número de casos de giardiose vem crescendo nos últimos anos em todas as categorias sócioeconômicas e que $22,7 \%$ do total dos casos ocorreram em pessoas envolvidas de alguma forma com o ambiente-creche (funcionários e familiares das crianças atendidas em creches). Estes autores acentuam a necessidade da realização de estudos longitudinais para explicar as tendências atuais em giardiose. Acresce que, no Brasil, faltam informações sobre a avaliação prospectiva da ocorrência de $G$. duodenalis entre crianças freqüentando creches.

Birkhead e col ${ }^{6}$ relataram que a giardiose é mais freqüente no sexo masculino. Neste estudo, não houve diferença significativa entre os sexos. O fato de freqüentar uma creche, por si só, poderia estar influenciando as taxas observadas e assim, meninos e meninas, a partir 
Franco RMB, Cordeiro NS. Giardiose e criptosporidiose em creches no municipio de Campinas, SP. Revista da Sociedade Brasileira de Medicina Tropical 29:585-591, nol'-dez, 1996.

da admissão na creche, experimentariam o mesmo risco de aquisição desta protozoose. Vários autores $>232933$ documentaram a maior ocorrência de giardiose entre as crianças que freqüentam creches.

C. parum tem distribuição cosmopolita, a prevalência desta parasitose situa-se em torno de 3,2\%-16,7\% para os países em desenvolvimento e as taxas de infecção são maiores em crianças com menos de 3 anos de idade ${ }^{1 .}$.

A positividade para criptosporidiose observada neste estudo $(6,4 \%)$, somada a outras descritas no país, como em Fortaleza $(8,0 \%)^{37}$, São Paulo $(17,0 \%)^{20}$, Recife $(6,4 \%)^{30} \mathrm{e}$ Belém $(5,2 \%)^{19}$ vem ressaltar que este coccídio é bastante freqüente em nosso meio, principalmente em crianças apresentando diarréia. A maioria dos levantamentos, no entanto, se referem a indivíduos sintomáticos, em atendimento ambulatorial ${ }^{20}{ }^{30}$. Estudos sobre a prevalência da criptosporidiose na população infantil, presumivelmente imunocompetente, freqüentando creches, recebeu menor atenção dos pesquisadores no Brasil. Este segmento populacional é de especial interesse pois, pelo fato de freqüentarem creches, estào mais expostas ao risco de aquisição de enteropatógenos.

Entre as crianças examinadas, C. parvum foi prevalente nos primeiros meses de vida (6 a 12 meses) o que coincide com as observaçôes de outros autores, tanto no Brasil $^{20}$ como no exterior ${ }^{22}{ }^{32}$. Crianças nesta faixa etária apresentaram maiores taxas de infecção, fato surpreendente se considerarmos que os lactentes incluídos na pesquisa estavam sob aleitamento materno parcial, o que poderia conferir alguma proteçào à criança ${ }^{10}$. É importante ressaltar que, conforme relatos dos responsáveis pelas crianças, estas não mostraram indícios de imunocomprometimento.

Quando levadas em consideração as características epidemiológicas das crianças portadoras de criptosporidiose, merece destaque a observação que a ocupação dos pais incluía profissionais da Área de Saúde do hospital universitário $(73,6 \%)$, muitas vezes em contato com pacientes imunocomprometidos. Transmissão de criptosporidiose em adultos, engajados'em atividade profissional como veterinários e enfermeitas, tem sido documentada na literatura $^{511}$. Outro ponto a ser ressaltado é o fato que os casos positivos para C. parum haviam apresentado recentemente, infecção concomitante das vias áereas superiores. Todavia, se a infecção respiratória predispōe à aquisição de C. paruum ou, se este protozoário, diminuindo a resistência do indivíduo, facilitaria a colonização por outros patógenos, é uma questão a ser esclarecida.

Quanto às demais parasitoses, os achados obtidos neste estudo confirmam o consenso de que as helmintoses intestinais tem baixa prevalência ${ }^{3+}$ em crianças entre 2 a 60 meses de idade, exceto se as condições sócioeconômicas são precárias. Pobreza e nível sócio-econômico são fatores críticos para aquisição de parasitoses na tenra idade ${ }^{34}$

\section{SUMMARY}

The prevalence of the Giardia duodenalis and Cryptosporidium parvum infections were studied in children 2-60 months old $(n=310)$ grouped to sex and age, enrolled in 8 day-care centers. Giardiosis was found in 42 (13.5\%) of the children and, was most frequent in the age group corresponding to 1924 months old although children at the first six months of life also presented positive faecal samples. Statistical analysis indicated no association between sex $(p>0,05)$ and prevalence for Giardia infection. C. parvum u'as detected in $20(6.4 \%)$ of the children. Children seven to twelne months old sbowed the greatest pretalence of this parasite. There were not oocysts in the stools from the children 25-30 months and from those older than 36 months. Oocysts were detected in 4 children from the $0-6$ months old group. There uas no significatize difference on sex $(p=0,09)$ and day-care facility $(p=0,068)$ for $\mathrm{C}$. parvum infection while age was associated with infection $(p=0,004)$. Epidemiological aspects were studied by familiar inquiry, by parasitological examinations of stools from the contact-members of eacb case and from animals present at the residence. Pretalence for $\mathrm{C}$. parvum was higher when at least one of the parents was a professional in the Human Health Centers ( $73.6 \%$ positives), when there was report of concomitant disease (52.6\% positives) and when diarrboea was the main symptom at the collection of the faecal specimens ( $78.9 \%$ positives). Faecal examinations of the domestic animals were negatives for these two parasites.

Key-uords: Criptosporidiosis. Giardiosis. Children. Day-care facilities.

\section{AGRADECIMENTOS}

Aos responsáveis pelas creches, pela colaboração. Ao estatístico José Luis Llanos 
Carrillo (Research International do Brasil), pela análise dos dados.

\section{REFERÊNCIAS BIBLIOGRÁFICAS}

1. Addiss DG, Davis JP, Roberts JM, Mast EE Epidemiology of Giardiasis in Wisconsin: increasing incidence of reported cases and unexplained seasonal trends. American Journal of Tropical Medicine and Hygiene 47: 13-19, 1992.

2. Addiss DG, Stewart JM, Finton RJ, Wahlquirst SP, Williams RM, Dickerson JW, Spencer HC, Juranek DD. Giardia lamblia and Cryptosporidium infections in child day-care centers in Fulton County, Georgia. Pediatric Infectious Diseases Journal 10:907-11, 1991.

3. Alpert G, Bell LM, Kirpatrick CE, Budnick LD, Campos JM, Friedman HM, Plotkin SA. Outbreak of cryptosporidiosis in a day-care center. Pediatrics 77: 152-7, 1986.

4. Bartlett AV, Moore M, Gay GW, Starko KM, Erben JJ, Meredith BA Diarrheal illness among infants and toddlers in day-care centers. I. Epidemiology and pathogens. Journal of Pediatric 107: 495-502, 1985.

5. Baxby D, Hart CA, Taylor C. Human cryptosporidiosis: a possible case of hospital cross infection. British Medical Journal 287: 176061, 1983.

6. Birkhead G, Vogt RL. Epidemiologic surveillance for endemic Giardia lamblia infection in Vermont. American Journal Epidemiology 129: 762-68, 1989.

7. Black RE, Dukes AC, Sinclair SP, Wells JG. Giardiasis in day-care centers: evidence of person-to-person transmission. Pediatrics 60: 486-91, 1977.

8. Blewett DA, Wright SE, Casemore DP, Booth N, Jones C. Infective dose size studies on Cryptosporidium paruum using gnotobiotic lambs. Water Science Technology 27: 61-64, 1993.

9. Cardoso G de Sá, Santana AD, Aguiar CP. Prevalência e aspectos epidemiológicos da giardíase em creches no Município de Aracajú, CE, Brasil. Revista da Sociedade Brasileira de Medicina Tropical 28:25-31, 1995.

10. Casemore DP. Epidemiological aspects of human cryptosporidiosis. Epidemiology and Infection 104: 1-28, 1990.

11. Casemore DP, Gardner CA, O'Mahony C. Cryptosporidial infection, with special reference to nosocomial transmission of Cryptosporidium paruum: a review. Folia Parasitologica 41: 17-22, 1994.

12. Coelho KIR, Maeda SA, Marques MEA. Intestinal cryptosporidiosis.Association with Pneumocystis carinii, cytomegalovirus and Candida sp. infections. Revista do Instituto de Medicina tropical de São Paulo 29:323-26, 1987.

13. Cordell RL, Addiss DG. Cryptosporidiosis in child care settings: a review of the literature and recommendations for prevention and control. Pediatric Infectious Diseases Journal 13: 310-17, 1994

14. Current WL, Garcia LS. Cryptosporidiosis. Clinical Microbiology Reviews 4: 325-58, 1991.

15. DuPont HE, Pickering LH. Infections of the gastrointestinal tract. Epidemiology, Microbiology and Clinical Features. Plenum. New York. 1980.

16. Henriksen A, Polhenz JFL. Staining of cryptosporidia by a modified Ziehl-Neelsen technique. Acta Veterinaria Scandinavia 22: 59496, 1981.

17. Janoff EN, Mead PS, Mead JR, Echeverria P, Bodhidatta L, Bhaiubulaya M, Sterling, CR, Taylor DN. Endemic Cryptosporidium and Giardia lamblia infections in a Thai orphanage.American Journal of Tropical Medicine and Hygiene 43: 24856, 1990.

18. Koch KJ, Phillips DJ, Aber RC, Current WL. Cryptosporidiosis in hospital personnel: evidence for person-to-person transmission. Annals of Internals Medicine 102: 593-96, 1985.

19. Loureiro ECB, Linhares A da Costa, Mata L. Criptosporidiose em crianças de 1 a 2 anos de idade, com diarréia aguda em Belém, Pará, Brasil. Memórias do Instituto Oswaldo Cruz 84: 117-122, 1989.

20. Mangini ACS, Dias RMD, Grisi SJFE, Escobar AMV, Torres DMAGV, Zuba, IPR, Quadros CMS, Chieffi PP. Parasitismo por Cryptosporidium sp. em crianças com diarréia aguda. Revista Instituto de Medicina tropical São Paulo 34: 341-45, 1992.

21. Marwick C; Simmons K. Changing childhood disease pattern linked with day-care boom. Journal American Medicine Association 251: 1245-51, 1984.

22. Mathan MM, Venkatesan S, George R, Mathew M, Mathan VI. Cryptosporidium and diarrhea in Southern Indian children. Lancet 2: 1172-75, 1985.

23. Novotny TE, Hopkins RS, Shillam P, Janoff EN. Prevalence of Giardia lamblia and risk factors 
Franco RMB, Cordeiro NS. Giardiose e criptosporidiose em creches no municipio de Campinas, SP. Revista da Sociedade Brasileira de Medicina Tropical 29:585-591, nov-dez, 1996.

for infection among children attending day-care facilities in Denver. Public Health Reports 105:7275,1990

24. Oliveira, A. Protozooses intestinais associadas à diarréia aguda infantil. Tese de Mestrado. Universidade Federal de Pernambuco, Recife, 1991.

25. Pickering LK, Engelkirk PG. Giardia lamblia. Pediatric Clinics of North America 35: 565-77, 1988.

26. Pickering LK. Woodward WE, DuPont HL, Sullivan P. Occurrence of Giardia lamblia in children in day-care centers. Journal of Pediatric 104:522-26, 1984.

27. Rentdorff RC. The experimental transmission of human intestinal protozoan parasites. II. Giardia lamblia cysts given in capsules.American Journal of Hygiene 59: 209-20, 1954.

28. Ritchie LS. An ether sedimentation technique for routine stool examinations. Bulletin of US Army Medical Departament 8:326, 1948.

29. Sealy DP. Schuman SH. Endemic Giardiasis and Day-care. Pediatrics, 72: 1154-58, 1983.

30. Silva GAP da, Magalhães M, Oliveira AMA, Melo UM. Diarréia aguda do lactente associado ao Cryptosporidium sp. na cidade do Recife. Jornal de Pediatria (Rio) 70:44-47, 1994.

31. Sullivan P, Woodward WE, Pickering LK, Du Pont H. Longitudinal study of occurrence of diarrheal disease in day-care centers. American Journal Public Health 74: 987-91, 1984.

32. Taylor JP, Perdue JN, Dindley D, Gustafson TL, Patterson M, Redd LA. Cryptosporidiosis outbreak in a day-care center.American Journal Diseases of Childhood 139: 1023-25, 1985.

33. Thompson SC. Giardia lamblia in children and the child care setting: A review of the literature. Journal Pediatric Child Health 30: 202-9, 1994.

34. Thompson SC. Infectious diarrhoea in children: controlling transmission in the child care setting. Journal Pediatric Child Health 30: 210-19, 1994.

35. Torres DMAGV, Chieffi PP, Costa WAC, Kudzielics E. Giardiase em creches mantidas pela Prefeitura do Município de São Paulo. Revista do Instituto de Medicina Tropical de São Paulo 33: 137-42, 1991.

36. Waldman E, Tzipori S, Forsyth JRL. Separation of cryptosporidium species oocysts from feces by using a Percoll discontinuous density gradient. Journal of Clinical Microbiology 23: 199-200, 1986.

37. Weikel CS, Johnston LI, Souza MA, Guerrant RL. Cryptosporidiosis in northeastern Brazil: association with sporadic diarrhoea. Journal of Infectious Diseases 151:963-5,1985. 